\title{
Random Attractor of Reaction-Diffusion Hopfield Neural Networks Driven by Wiener Processes
}

\author{
Xiao Liang $\mathbb{D},{ }^{1,2}$ Linshan Wang $\mathbb{D}^{3}$, and Ruili Wang $\mathbb{D}^{2}$ \\ ${ }^{1}$ College of Mathematics and Systems Science, Shandong University of Science and Technology, Qingdao 266590, China \\ ${ }^{2}$ Beijing Institute of Applied Physics and Computational Mathematics, Beijing 100094, China \\ ${ }^{3}$ School of Mathematical Sciences, Ocean University of China, Qingdao 266100, China
}

Correspondence should be addressed to Linshan Wang; wangls@ouc.edu.cn and Ruili Wang; wang_ruili@iapcm.ac.cn

Received 12 July 2017; Accepted 6 November 2017; Published 16 January 2018

Academic Editor: Fazal M. Mahomed

Copyright (C) 2018 Xiao Liang et al. This is an open access article distributed under the Creative Commons Attribution License, which permits unrestricted use, distribution, and reproduction in any medium, provided the original work is properly cited.

\begin{abstract}
This paper studies the global existence and uniqueness of the mild solution for reaction-diffusion Hopfield neural networks (RDHNNs) driven by Wiener processes by applying a Schauder fixed point theorem and a priori estimate; then the random attractor for this system is also studied by constructing proper random dynamical system.
\end{abstract}

\section{Introduction}

It is well known that the dynamics of Hopfield neural networks have been deeply investigated because they have been successfully employed in many areas such as pattern recognition, associate memory, and combinatorial optimization. The diffusion effect cannot be avoided in the neural networks when electrons are moving in asymmetric electromagnetic field. So, the dynamical behavior of reactiondiffusion Hopfield neural networks (RDHNNs, for short) has been receiving much attention, recently [1-11].

But in a more realistic model, in order to describe the propagation of an electric potential in a neuron, it is sensible to include some noise in the system. In fact, a neural network can be stabilized or destabilized by certain stochastic inputs. Many scholars have been devoted to the stochastic RDHNNs such as $[2,12,13]$. Giving a deep insight into these literatures, we will find that most of these literatures consider the RDHNNs with finite dimensional Wiener processes; there are few results on the RDHNNs driven by infinite dimensional Wiener processes. However, since the neurons can be regarded as long thin cylinders, which act like electrical cables, the infinite dimensional Wiener processes are more favorable than standard Brownian motion.

On the other hand, attractor plays an important role in the long time behavior for dynamical systems. The random attractor extends the concept of a strange attractor from deterministic to stochastic system. There has been great interest in random attractors for stochastic partial differential equations in recent decades. The random attractors are compact invariant random sets attracting all the orbits of attraction basin. They provide crucial geometric information about their asymptotic regime as $t \rightarrow \infty$. They can help us understand the chaotic behavior of the stochastic DRDHNNs and reduce the complexity, as well as providing the statistical properties of this system. When the global existence and uniqueness of the solution can be assured, many scholars pay much attention to the global stability, boundedness, and even synchronization of the RDHNNs $[1-3,11]$. However, to the best of our knowledge, there is no result on the attractor for RDHNNs, let alone random attractor for stochastic RDHNNs. We hope this work can lay a solid foundation for the future research.

So, this paper is devoted to the random attractor for the following RDHNNs driven by Wiener processes:

$$
\begin{array}{r}
\mathrm{d} u_{i}(t, \mathbf{x})=\left(\sum_{j=1}^{l} \frac{\partial}{\partial x_{j}}\left(G_{i j}(\mathbf{x}) \frac{\partial u_{i}}{\partial x_{j}}\right)-b_{i} u_{i}\right. \\
\left.+\sum_{j=1}^{n} c_{i j} f_{j}\left(u_{j}\right)+I_{\mathrm{i}}\right) \mathrm{d} t+\sigma_{i} \mathrm{~d} W_{i}(t, \mathbf{x}),
\end{array}
$$




$$
\begin{aligned}
& u_{i}(t, \mathbf{x})=0, \quad t \geq t_{0}, \quad \mathbf{x} \in \partial \mathcal{O}, \\
& u_{i}\left(t_{0}, \mathbf{x}\right)=\phi_{i}(\mathbf{x}), \quad \mathbf{x} \in \mathcal{O} \in \mathbb{R}^{l}, i=1,2, \ldots, n,
\end{aligned}
$$

where $u_{i}(t, \mathbf{x})$ denote the potential of the cell $i$ at $t$ and $\mathbf{x} \in \mathbb{R}^{l}$. $b_{i}$ are positive constants and denote the rate with which the $i$ th unit will reset its potential to the resting state in isolation when it is disconnected from the network and external inputs at $t$. $c_{i j}$ are connection weights of the neural network. $f_{i}$ are the active functions of the neural network, which are continuous. $\sigma_{i}$ are the intensity of the noise. $I_{i}$ denote the $i$ th component of an external input source introduced from outside the network to the $i$ th neuron, which are constant numbers. $\mathcal{O}$ denotes an open bounded and connected subset of $\mathbb{R}^{l}$ with a sufficiently regular boundary $\partial \mathcal{O} . \nabla$ is the gradient. Initial data $\phi_{i}$ are $\mathscr{F}_{0}$-measurable and belong to $L^{2}(\mathcal{O})$, a.e. $\omega \in \Omega$.

For convenience, we rewrite system (1) in the vector form

$$
\begin{aligned}
\mathrm{d} \mathbf{u}= & (\nabla \cdot(G(\mathbf{x}) \circ \nabla \mathbf{u})-\mathbf{B u}+\mathbf{C} \tilde{f}(\mathbf{u})+\mathbf{I}) \mathrm{d} t \\
& +\sigma \mathrm{d} \mathbf{W}, \\
\mathbf{u}(t, \mathbf{x})= & 0, \quad t \geq t_{0}, \mathbf{x} \in \partial \mathcal{O}, \\
\mathbf{u}\left(t_{0}, \mathbf{x}\right)= & \phi(\mathbf{x}),
\end{aligned}
$$

where $\mathbf{C}=\left(c_{i j}\right)_{n \times n}, \sigma=\operatorname{diag}\left\{\sigma_{1}, \sigma_{2}, \ldots, \sigma_{n}\right\} \mathbf{I}=\left(I_{1}, I_{2}\right.$, $\left.\ldots, I_{n}\right)^{T}, \mathbf{u}=\left(u_{1}, u_{2}, \ldots, u_{n}\right)^{T}, \nabla \mathbf{u}=\left(\nabla u_{1}, \nabla u_{2}, \ldots, \nabla u_{n}\right)^{T}$, $\mathbf{W}=\left(W_{1}, W_{2}, \ldots, W_{n}\right)^{T}, \widetilde{\mathbf{f}}(\mathbf{u})=\left(f_{1}\left(u_{1}\right), f_{2}\left(u_{2}\right), \ldots, f_{n}\left(u_{n}\right)\right)^{T}$ is the diagonal map, $\nabla$ is the gradient operator, $\mathbf{B}=$ $\operatorname{diag}\left(b_{1}, b_{2}, \ldots, b_{n}\right), \boldsymbol{\phi}(\mathbf{x})=\left(\phi_{1}(\mathbf{x}), \phi_{2}(\mathbf{x}), \ldots, \phi_{n}(\mathbf{x})\right)^{T}, \mathbf{G}(\mathbf{x})=$ $\left(G_{i j}\right)_{n \times l}, \mathbf{G} \circ \nabla \mathbf{u}=\left(G_{i j}\left(\partial u_{i} / \partial x_{j}\right)\right)_{n \times l}$ is the Hadamard product between matrix $\mathbf{G}$ and $\nabla \mathbf{u}$, and $\nabla \cdot$ is the divergence operator.

We will also use the following notations in the paper.

(i) $(\Omega, \mathscr{F}, \mathbb{P})$ is a complete probability space with filtration $\left\{\mathscr{F}_{t}\right\}_{t \geq 0}$ satisfying the usual conditions (see $[1,2,14-18])$.

(ii) $\mathbf{W}(t, \mathbf{x})=\mathbf{W}(t, \mathbf{x})(\omega)$ is a space-time Wiener process with values in the separable Hilbert space $K$ with $E \mathbf{W}=\mathbf{0}$ and $E(\mathbf{W}, \mathbf{u})_{K}(\mathbf{W}, \mathbf{v})_{K}=(t \wedge s)(Q \mathbf{u}, \mathbf{v})_{X}$, $\forall s, t \geq 0, \mathbf{u}, \mathbf{v} \in X,(\cdot, \cdot)_{X}$ denotes the inner product of $X, \mathbf{W}(t, \mathbf{x})=\sum_{n=1}^{\infty} \sqrt{\alpha_{n}} \beta_{n}(t) e_{n}(\mathbf{x})$, where $t \wedge$ $s=\min \{t, s\}$ and the Hilbert-Schmidt operator $Q$ is a positive definite, nuclear, symmetric, self-adjoint operator having a finite trace $\operatorname{tr} Q \triangleq \sum_{n=1}^{\infty} \alpha_{n}<+\infty$ with eigenvalues $\alpha_{n},\left\{e_{n}\right\}_{n=1}^{\infty}$ is an orthogonal basis of $X$, and $\left\{\beta_{n}\right\}_{n=1}^{\infty}$ is a sequence of mutually independent standard Brownian motions in $(\Omega, \mathscr{F}, \mathbb{P})$ (see $[2,14])$.

(iii) $\mathscr{L}_{2}\left(X_{0}, U\right)$ is the space of all Hilbert-Schmidt operators from $X_{0} \triangleq Q^{1 / 2}(X)$ into $U$; when equipped with the norm $\|\Phi\|_{2} \triangleq \sqrt{\operatorname{tr}\left(\Phi Q \Phi^{*}\right)}$ it becomes a Hilbert space, where $\Phi \in \mathscr{L}_{2}\left(X_{0}, U\right) ; \Phi^{*}$ denotes the adjoint of $\Phi$.

\section{Preliminaries and Notations}

In this paper, we introduce the following Hilbert spaces: $U=$ $\left\{L^{2}(\mathcal{O})\right\}^{n}$ and $V=\left\{H^{1}(\mathcal{O})\right\}^{n}$; according to [14, 19-21], $V \subset$ $U=U^{\prime} \subset V^{\prime} ; U^{\prime}, V^{\prime}$ denote the dual of the spaces $U$, $V$ respectively, the injection continues, and the embedding is compact; $\|\cdot\|,\|\cdot\|$ represent the usual norm in $U, V$, respectively. Let us define the operator as follows:

$$
\begin{aligned}
A: \Pi(A) & \in U \longrightarrow U \\
A \mathbf{u} & =\nabla \cdot(\mathbf{G} \circ \nabla \mathbf{u})-\mathbf{B u} \mathbf{u}
\end{aligned}
$$

and $\Pi(A)$ is the domain of $A$ defined as $\Pi(A)=\left\{H^{2}(\mathcal{O})\right\}^{n} \cap$ $\left\{H_{0}^{1}(\mathcal{O})\right\}^{n} . A$ is the infinitesimal generator of an analytic semigroup $T(t)$.

Defining the Nemytskii operator as follows:

$$
\mathbf{f}(\mathbf{u})(\mathbf{x})=\tilde{f}(\mathbf{u}(\mathbf{x})), \quad \forall \mathbf{x} \in \mathcal{O} .
$$

With these notations, we rewrite system (2) in the more abstract form

$$
\begin{aligned}
\mathrm{d} \mathbf{u} & =(A \mathbf{u}+\mathbf{C f}(\mathbf{u})+\mathbf{I}) \mathrm{d} t+\sigma \mathrm{d} \mathbf{W}, \\
\mathbf{u}\left(t_{0}\right) & =\phi .
\end{aligned}
$$

We recall that

$$
\mathbf{W}_{\mathbf{A}}(t)=\sigma \int_{-\infty}^{t} e^{A(t-s)} \mathrm{d} \mathbf{W}(s)
$$

is the solution of the Ornstein-Uhlenbeck process

$$
\mathrm{d} \mathbf{u}=A \mathbf{u d} t+\sigma \mathrm{d} \mathbf{W}
$$

The regularity of (6) has been proved in $[14,22]$ and $\mathbf{W}_{\mathbf{A}}(t)$ has a $\alpha$-Hölder continuous version with respect to $t, \alpha<1 / 4$. Furthermore, by the law of large number

$$
\begin{aligned}
& \lim _{t \rightarrow \pm \infty} \frac{\left\|\mathbf{W}_{\mathbf{A}}(t)\right\|}{|t|}=0, \\
& \lim _{t \rightarrow \pm \infty} \frac{\left\|\ln \mathbf{W}_{\mathbf{A}}(t)\right\|}{|t|}=0,
\end{aligned}
$$

a.s.

(H1) We assume that $\|\mathbf{f}(\mathbf{u})\| \leq k_{1}+k_{2}\|\mathbf{u}\|,\|\mathbf{f}(\mathbf{u})-\mathbf{f}(\mathbf{v})\| \leq$ $k_{3}\|\mathbf{u}-\mathbf{v}\|, \forall \mathbf{u}, \mathbf{v} \in U$.

(H2) We assume that there exists a positive number $\beta(\mathcal{O})$ such that the following Poincaré inequality is valid:

$$
\|\mathbf{u}\| \leq \beta^{-1}\|\mathbf{u}\|, \quad \forall \mathbf{u} \in V
$$

(H3) We assume that there exists $\alpha>0$ such that $G_{i j}(\mathbf{x}) \geq$ $\alpha / n l, i=1,2, \ldots, n$.

(H4) Let $k_{4}>\sqrt{2} k_{2} k_{5}$, with $k_{4}=\min \left\{b_{1}, b_{2}, \ldots, b_{n}\right\}$ and $k_{5}=\max \left\{\left|c_{i j}\right|\right\}$. 
We also need the following propositions in the following sections.

Proposition 1. Consider the following equation:

$$
\begin{aligned}
\frac{\mathrm{d}}{\mathrm{d} t} \mathbf{u} & =A \mathbf{u}, \quad t \geq 0, \\
\mathbf{u}(0) & =\phi
\end{aligned}
$$

Suppose (H2), (H3) hold; let $\mathbf{u}(t)=T(t) \phi$ denote the mild solution of (10); then $T(t)$ is a contraction map in $U$.

Proof. We recall that the solution of this linear equation is $\mathbf{u}(t)=e^{A t} \boldsymbol{\phi}$, so $T(t)=e^{A t}$. Now we take the inner product of (10) with $\mathbf{u}(t)$ in $U$, by employing the Gaussian theorem and condition $(\mathrm{H} 3)$, we get

$$
(A \mathbf{u}, \mathbf{u}) \leq-\alpha\|\mathbf{u}\|^{2}-k_{4}\|\mathbf{u}\|^{2}, \quad \forall \mathbf{u} \in V
$$

where $(\cdot, \cdot)$ is the inner product in $U$ (see [1-3]), and we also have

$$
\frac{1}{2} \frac{\mathrm{d}}{\mathrm{d} t}\|\mathbf{u}(t)\|^{2}+\alpha\|\mathbf{u}(t)\|^{2}+k_{4}\|\mathbf{u}\|^{2} \leq 0 .
$$

By $(\mathrm{H} 2)$, one obtains

$$
\frac{\mathrm{d}}{\mathrm{d} t}\|\mathbf{u}(t)\|^{2}+2\left(\alpha \beta^{2}+k_{4}\right)\|\mathbf{u}(t)\|^{2} \leq 0
$$

By Gronwall-Bellman inequality, we have

$$
\|\mathbf{u}(t)\|^{2} \leq e^{-2\left(\alpha \beta^{2}+k_{4}\right) t}\|\phi\|^{2}
$$

by the definition of $\|T(t)\|$ and uniform boundedness principle, we have

$$
\|T(t)\| \leq e^{-\left(\alpha \beta^{2}+k_{4}\right) t} \leq 1 .
$$

So $T(t)$ is a contraction map.

Remark 2 (see [14]). Let $T(t)$ be a strongly continuous semigroup and $A$ be the generator of $T(t)$; then we have

$$
\begin{aligned}
T\left(t_{1}\right) \phi-T\left(t_{2}\right) \phi & =\int_{t_{2}}^{t_{1}} T(s) A \phi \mathrm{d} s \\
& =\int_{t_{2}}^{t_{1}} A T(s) \phi \mathrm{d} s, \quad \forall \phi \in \Pi(A) .
\end{aligned}
$$

Proposition 3 (see [2]). Let $\Psi=\left(\psi_{i j}\right)_{n \times n}$ and $\mathbf{u}=$ $\left(u_{1}, u_{2}, \ldots, u_{n}\right)^{T}$; then $|\Psi \mathbf{u}|_{\mathbb{R}^{n}} \leq \sqrt{n}\|\Psi\|_{F}\|\mathbf{u}\|_{\mathbb{R}^{n}}$, where $\|\mathbf{u}\|_{\mathbb{R}^{n}}$ represents the usual norm of $\mathbb{R}^{n}$ and $\|\Psi\|_{F}$ represents the Frobenius norm of a matrix $\Psi \in \mathbb{R}^{n \times n}$; that is, $\|\Psi\|_{F}=$ $\left(\sum_{i=1}^{n} \sum_{j=1}^{n} \psi_{i j}^{2}\right)^{1 / 2}$.

Let $(X ; d)$ be a complete separable metric space. We shall recall the notions of random dynamical system and random attractor.
Definition 4 (see [22]). Let $(X, d)$ be a complete separable metric space. A metric dynamical system (MDS) $\theta \triangleq$ $\left(\Omega, \mathscr{F}, \mathbb{P},\left\{\theta_{t}, t \in R\right\}\right)$ is a family of measure-preserving transformations $\theta_{t}: \Omega \rightarrow \Omega, t \in \mathbb{R}$ such that

(i) $\theta_{0}=i d, \theta_{t} \cdot \theta_{s}=\theta_{t+s}$ for all $t, s \in \mathbb{R}$; $\bullet$ is the composition operator;

(ii) the map $(t, \omega) \rightarrow \theta_{t}(\omega)$ is $\mathscr{B}(\mathbb{R}) \otimes \mathscr{F}$ measurable, and $\theta_{t} \mathbb{P}=\mathbb{P}$ for all $t \in \mathbb{R}$, where $\mathscr{B}(\mathbb{R})$ is the Borel $\sigma$ field of $\mathbb{R}$.

Definition 5 (see [22-25]). A random dynamical system (RDS) over $\theta_{t}$ is a measurable map $\varphi$, such that

(1) $\varphi(0, \omega) x=x, x \in X, \omega \in \Omega$;

(2) $\varphi(s+t, \omega) x=\varphi\left(s, \theta_{t} \omega\right) \cdot \varphi(t, \omega) x, s, t \in \mathbb{R}, x \in X$, $\omega \in \Omega$;

(3) $(t, x) \rightarrow \varphi(t, \omega) x$ is continuous a.e. $\omega \in \Omega$;

(4) $\omega \rightarrow \boldsymbol{\varphi}(t, \omega) x$ is $\mathscr{F}$-measurable for all $(t, x) \in \mathbb{R} \times X$.

Definition 6 (see $[26,27])$. A stochastic flow is a family of mappings $S(t, s ; \omega): X \rightarrow X,-\infty<s \leq t<\infty$, parameterized by $\omega$, such that

$$
(t, s, x, \omega) \longrightarrow S(t, s ; \omega) x
$$

is $\mathscr{B}(\mathbb{R}) \otimes \mathscr{B}(\mathbb{R}) \otimes \mathscr{B}(X) \otimes \mathscr{F}$-measurable and

$$
\begin{aligned}
S(t, r ; \omega) \bullet S(r, s ; \omega) x & =S(t, s ; \omega) x, \\
s \leq r \leq t, x \in X, & \\
S(t, s ; \omega) x & =S\left(t-s, 0 ; \theta_{s} \omega\right) x, \quad \omega \in \Omega .
\end{aligned}
$$

$S$ is said to be a continuous stochastic flow, if $x \rightarrow S(t, s ; \omega) x$ is continuous.

Definition 7 (see $[28,29]$ ). A map $K: \Omega \rightarrow 2^{X}$ is said to be a closed random set if $K(\omega)$ is closed for a.e. $\omega \in \Omega$ and the map $\omega \rightarrow \operatorname{dist}(x, K(\omega))$ is a.e. measurable for all $x \in X$; dist denotes the Hausdorff semidistance defined as $\operatorname{dist}(x, \mathbb{A})=$ $\inf _{y \in \mathbb{A}} d(x, y)$ and $\operatorname{dist}(\mathbb{A}, \mathbb{B})=\sup _{x \in \mathbb{A}} d(x, \mathbb{B}), \mathbb{A}, \mathbb{B} \in 2^{X}$.

Definition 8 (see $[29,30]$ ). A map $K: \Omega \rightarrow 2^{X}$ is said to be a compact random set if $K(\omega)$ is compact for a.e. $\omega \in \Omega$ and the map $\omega \rightarrow \operatorname{dist}(x, K(\omega))$ is a.s. measurable.

Definition 9 (see $[31,32]$ ). A random set $K(\omega)$ is called an absorbing set in $X$, if for all $\mathbb{B} \in X$ and a.e. $\omega \in \Omega$, there exists $t_{\mathbb{B}}(\omega)>0$ such that $t \geq t_{\mathbb{B}}(\omega)>0$

$$
\boldsymbol{\varphi}\left(t, \theta_{-t} \omega\right) \mathbb{B} \subset K(\omega) \text {. }
$$

Lemma 10 (Schauder fixed point theorem [33-38]). If $\mathbb{B}$ is a closed bounded convex subset of a space $X$ and $\mathfrak{Q}: \mathbb{B} \rightarrow \mathbb{B}$ is completely continuous, then $\mathbf{Q}$ has a fixed point in $\mathbb{B}$.

Lemma 11 (see $[39,40]$ ). Suppose $\boldsymbol{\varphi}$ is a RDS on a Polish space $X$, and there exists a compact set $K(\omega)$ absorbing every bounded deterministic set $\mathbb{B} \in X$. Then the set

$$
\mathfrak{A}(\omega)=\overline{\bigcup_{\mathbb{B} \in X} \bigcap_{T \geq 0} \overline{\bigcup_{t \geq T} \phi\left(t, \theta_{-t} \omega\right) \mathbb{B}}}
$$

is a random attractor for $\boldsymbol{\varphi}$. 


\section{Existence and Uniqueness of the Solutions}

Let

$$
\mathbf{v}(t)=\mathbf{u}(t)-\mathbf{W}_{\mathbf{A}}(t),
$$

where $\mathbf{W}_{\mathbf{A}}(t)$ has been defined in the previous section. Then, from (5) and (6), $\mathbf{v}(t)$ satisfies the equation

$$
\begin{aligned}
\mathrm{d} \mathbf{v} & =\left(\mathbf{A v}+\mathbf{C f}\left(\mathbf{v}+\mathbf{W}_{\mathbf{A}}\right)+\mathbf{I}\right) \mathrm{d} t, \\
\mathbf{v}\left(t_{0}\right) & =\psi,
\end{aligned}
$$

where $\psi=\phi-\mathbf{W}_{\mathbf{A}}\left(t_{0}\right)$. Let us rewrite (23) in the integral form

$$
\mathbf{v}(t, \omega)=T(t) \psi+\int_{t_{0}}^{t} T(t-s)\left(\mathbf{C f}\left(\mathbf{v}+\mathbf{W}_{\mathbf{A}}\right)+\mathbf{I}\right) \mathrm{d} s .
$$

Definition 12. If $\mathbf{v}$ satisfies (23), we say that the $\mathbf{u}(t)$ is a mild solution of (1).

Let $X_{T^{*}} \triangleq C\left(\left[t_{0}, t_{0}+T^{*}\right] ; U\right)$, when equipped with the norm $\|\mathbf{u}\|_{X_{T^{*}}}=\left(\sup _{t \in\left[t_{0}, t_{0}+T^{*}\right]}\|\mathbf{u}(t)\|^{2}\right)^{1 / 2}$, it becomes a Banach space. Let

$$
\begin{aligned}
& \sum\left(m, T^{*}\right)=\left\{\mathbf{v} \in C\left(\left[t_{0}, t_{0}+T^{*}\right] ; U\right):\|\mathbf{v}(t)\| \leq m \forall t\right. \\
& \left.\quad \in\left[t_{0}, t_{0}+T^{*}\right]\right\}
\end{aligned}
$$

and consider an initial data $\psi$ which is $\mathscr{F}_{0}$-measurable and belong to $U$, a.e. $\omega \in \Omega$. From now on, we are going to discuss mild solution of equation (22) by Schauder fixed point method in the space $X_{T^{*}}$ for some $T^{*}>0$.

Theorem 13. Assume that $\psi$ is $\mathscr{F}_{0}$-measurable and belongs to $U$, a.e. $\omega \in \Omega$. If conditions (H1)-(H3) hold, then for any $\|\psi\|<$ $m$, there exists a stopping time $T^{*}>0$, such that (1) has a mild solution in $\Sigma\left(m, T^{*}\right)$.

Proof. We split our proof into the following steps.

Step 1. $\Sigma\left(m, T^{*}\right)$ is a nonempty closed bounded convex set.

Let $\mathbf{v}_{1}, \mathbf{v}_{2}$ belong to $\Sigma\left(m, T^{*}\right)$; then for $0 \leq \lambda \leq 1$

$$
\begin{aligned}
\left\|\lambda \mathbf{v}_{1}+(1-\lambda) \mathbf{v}_{2}\right\|_{X_{T^{*}}} & \leq \lambda\left\|\mathbf{v}_{1}\right\|_{X_{T^{*}}}+(1-\lambda)\left\|\mathbf{v}_{2}\right\|_{X_{T^{*}}} \\
& \leq m
\end{aligned}
$$

so $\lambda \mathbf{v}_{1}+(1-\lambda) \mathbf{v}_{2} \in \Sigma\left(m, T^{*}\right)$; hence $\Sigma\left(m, T^{*}\right)$ is a convex set.

Step 2. $\mathscr{T}$ maps $\Sigma\left(m, T^{*}\right)$ into $\Sigma\left(m, T^{*}\right)$.

Take any $\mathbf{v}$ in $\Sigma\left(m, T^{*}\right)$ and define $\mathbf{z}=\mathscr{T} \mathbf{v}$ by

$$
\mathbf{z}(t)=T(t) \psi+\int_{t_{0}}^{t} T(t-s)\left(\mathbf{C} \mathbf{f}\left(\mathbf{v}+\mathbf{W}_{\mathbf{A}}\right)+\mathbf{I}\right) \mathrm{d} s .
$$

By the triangle inequality

$$
\begin{aligned}
\|\mathbf{z}\| \leq & \|T(t) \psi\| \\
& +\left\|\int_{t_{0}}^{t} T(t-s)\left(\mathbf{C f}\left(\mathbf{v}+\mathbf{W}_{\mathbf{A}}\right)+\mathbf{I}\right) \mathrm{d} s\right\| .
\end{aligned}
$$

By Proposition 1, it follows that

$$
\|\mathbf{z}(t)\| \leq\|\psi\|+\int_{t_{0}}^{t}\left\|\mathbf{C f}\left(\mathbf{v}+\mathbf{W}_{\mathbf{A}}\right)+\mathbf{I}\right\| \mathrm{d} s .
$$

By using Proposition 3 and norm inequality, as well as condition (H1), we have

$$
\begin{aligned}
& \left\|\mathbf{C} \mathbf{f}\left(\mathbf{v}+\mathbf{W}_{\mathbf{A}}\right)\right\| \leq \sqrt{n}\|\mathbf{C}\|_{F}\left\|\mathbf{f}\left(\mathbf{v}+\mathbf{W}_{\mathbf{A}}\right)\right\| \\
& \quad \leq \sqrt{n}\|\mathbf{C}\|_{F}\left(k_{1}+k_{2}\left\|\mathbf{v}+\mathbf{W}_{\mathbf{A}}\right\|\right) \\
& \leq \sqrt{n}\|\mathbf{C}\|_{F}\left(k_{1}+k_{2}\left(\|\mathbf{v}\|+\left\|\mathbf{W}_{\mathbf{A}}\right\|\right)\right) \\
& \quad \leq \sqrt{n}\|\mathbf{C}\|_{F}\left(k_{1}+k_{2}\|\mathbf{v}\|_{X_{T^{*}}}+k_{2}\left\|\mathbf{W}_{\mathbf{A}}\right\|_{X_{T^{*}}}\right) ;
\end{aligned}
$$

therefore

$$
\begin{aligned}
\|\mathbf{z}(t)\|_{X_{T^{*}}} \leq & \|\psi\| \\
& +\int_{t_{0}}^{t}\left(k_{5}\left\|\mathbf{W}_{\mathbf{A}}\right\|_{X_{T^{*}}}+k_{6}+k_{7}\|\mathbf{v}\|_{X_{T^{*}}}\right) \mathrm{d} s \\
\leq & \|\psi\|+\left(k_{6} \mu+k_{7}+k_{8} m\right) t,
\end{aligned}
$$

where $\mu=\left\|\mathbf{W}_{\mathbf{A}}\right\|_{X_{T^{*}}}, k_{6}=\sqrt{n} k_{2}\|\mathbf{C}\|_{F}, k_{7}=\|\mathbf{I}\|+\sqrt{n}\|\mathbf{C}\|_{F} k_{1}$, and $k_{8}=\sqrt{n} k_{2}\|\mathbf{C}\|_{F}$.

Hence $\|\mathbf{z}(t)\| \leq m$ for all $t \in\left[t_{0}, t_{0}+T^{*}\right]$, provided that

$$
\|\psi\|+\left(k_{6} \mu+k_{7}+k_{8} m\right)\left(t-t_{0}\right)<m .
$$

It is clear that, for any $\|\psi\|<m$, there exists a $T^{*}$ satisfying (31).

Step 3. $\mathscr{T}$ is a equicontinuous map of $\Sigma\left(m, T^{*}\right)$.

Let $t_{1}, t_{2}>0$, then by Remark 2 and Propositions 1 and 3 , we have

$$
\begin{aligned}
\left\|\mathscr{T} \mathbf{v}\left(t_{1}\right)-\mathscr{T} \mathbf{v}\left(t_{2}\right)\right\| & \\
= & \left\|\left(T\left(t_{1}\right)-T\left(t_{2}\right)\right) \psi\right\| \\
& +\left\|\int_{t_{2}}^{t_{1}} T(t-s)\left(\mathbf{C} \mathbf{f}\left(\mathbf{v}+\mathbf{W}_{\mathbf{A}}\right)+\mathbf{I}\right) \mathrm{d} s\right\| \\
\leq & \int_{t_{2}}^{t_{1}}\|T(s) A \psi\| \mathrm{d} s \\
& +\int_{t_{2}}^{t_{1}}\left\|T(t-s)\left(\mathbf{C f}\left(\mathbf{v}+\mathbf{W}_{\mathbf{A}}\right)+\mathbf{I}\right)\right\| \mathrm{d} s \\
\leq & \int_{t_{2}}^{t_{1}}\|A \psi\| \mathrm{d} s+\int_{t_{2}}^{t_{1}}\left(\left\|\mathbf{C} \mathbf{f}\left(\mathbf{v}+\mathbf{W}_{\mathbf{A}}\right)\right\|+\|\mathbf{I}\|\right) \mathrm{d} s \\
\leq & \int_{t_{2}}^{t_{1}}\|A \psi\| \mathrm{d} s+\left(k_{6} \mu+k_{7}+k_{8} m\right)\left|t_{1}-t_{2}\right| \\
\leq & \left(\|A \psi\|+k_{6} \mu+k_{7}+k_{8} m\right)\left|t_{1}-t_{2}\right|,
\end{aligned}
$$

so $\mathscr{T} \Sigma\left(m, T^{*}\right)$ is an equicontinuous set. 
By the Arzelá-Ascoli theorem, for any bounded set $\mathscr{B} \subset$ $\Sigma\left(m, T^{*}\right)$, the closure of $\mathscr{T} \mathscr{B}$ is compact, so $\mathscr{T}$ is a completely continuous map, then by Steps 1-3 and Lemma 10, $\mathscr{T}$ has a fixed point in $\Sigma\left(m, T^{*}\right)$, which is a mild solution of (1).

Theorem 14. Suppose (H1)-(H3) hold, then for any $\|\psi\|<m$, (1) has at most one mild solution in $\left[t_{0}, t_{0}+T^{*}\right]$.

Proof. Let $\mathbf{v}_{1}, \mathbf{v}_{2} \in \Sigma\left(m, T^{*}\right)$ be two solutions of system (23) on $[0 ; T]$ with $\mathbf{v}_{1}(0)=\mathbf{v}_{2}(0)=\psi$, and set $\mathbf{z}_{i}=\mathscr{T} v_{i}, i=1,2$, and $\mathbf{z}=\mathbf{z}_{1}-\mathbf{z}_{2}$. Then

$$
\begin{aligned}
\mathbf{z}(t) & \\
\quad & T(t)\left(\mathbf{v}_{1}(0)-\mathbf{v}_{2}(0)\right) \\
& +\int_{t_{0}}^{t} T(t-s) \mathbf{C}\left(\mathbf{f}\left(\mathbf{v}_{1}+\mathbf{W}_{\mathbf{A}}\right)-\mathbf{f}\left(\mathbf{v}_{2}+\mathbf{W}_{\mathbf{A}}\right)\right) \mathrm{d} s .
\end{aligned}
$$

Following the method in Step 3 of Theorem 13, we have

$$
\|\mathbf{z}(t)\|_{X_{T^{*}}} \leq \sqrt{n}\|\mathbf{C}\|_{F} k_{3} T^{*}\left\|\mathbf{v}_{1}-\mathbf{v}_{2}\right\|_{X_{T^{*}}} .
$$

We take a stopping time $T^{*}$ such that

$$
\sqrt{n}\|\mathbf{C}\|_{F} k_{3} T^{*}<1
$$

which implies that $\mathbf{v}_{1}(t)=\mathbf{v}_{2}(t)$ and $t \in\left[t_{0}, t_{0}+T^{*}\right]$. By the combination of Theorem 13, there is one unique mild solution $\mathbf{u}(t)$ for (1).

Theorem 15. Suppose (H1)-(H3) hold. If $\mathbf{v} \in C\left(\left[t_{0}, t_{0}+T\right] ; U\right)$ is a solution of system (23), then

$$
\|\mathbf{v}(t)\| \leq\|\psi\|+\sqrt{\delta}
$$

for some constant $\mu_{\infty}>0$, where $\delta=e^{|\eta| T}\left(\|\mathbf{I}\|^{2}+\sqrt{n}\|\mathbf{C}\|_{F} k_{1}^{2}+\right.$ $\left.2 k_{2}^{2} \sqrt{n}\|\mathbf{C}\|_{F} \mu_{\infty}^{2}\right)$ and $\gamma=2 \alpha \beta^{2}+2 k_{4}-\sqrt{n}\|\mathbf{C}\|_{F}\left(2 k_{2}^{2}+1\right)-1$.

Proof. By employing the method in $[41,42]$, let $\left\{\psi^{m}\right\}_{m=1}^{\infty}$ be a sequence in $\left\{C^{\infty}(\mathcal{O})\right\}^{n}$ such that

$$
\psi^{m} \longrightarrow \psi \in U
$$

Let $\left\{\mathbf{W}_{\mathbf{A}}^{m}\right\}$ be a sequence of regular process such that

$$
\mathbf{W}_{\mathbf{A}}^{m}(t)=\int_{t_{0}}^{t} T(t-s) \mathrm{d} \mathbf{W}^{m}(s) \longrightarrow \mathbf{W}_{\mathbf{A}}(t)
$$

in $\left\{C\left(\left[t_{0}, t_{0}+T\right] \times \mathcal{O}\right)\right\}^{n}$ a.e. $\omega \in \Omega$.

Let $\mathbf{v}^{m}$ be the solution of

$$
\begin{aligned}
\mathbf{v}^{m}(t)= & T(t) \psi^{m} \\
& +\int_{t_{0}}^{t} T(t-s)\left(\mathbf{C f}\left(\mathbf{v}^{m}+\mathbf{W}_{A}^{m}\right)+\mathbf{I}\right) \mathrm{d} s .
\end{aligned}
$$

By using the method in the Theorem 13, it is easy to see that $\mathbf{v}^{m}$ does exist on an interval of time $\left[t_{0}, t_{0}+T^{m}\right]$ such that $T^{m} \rightarrow T^{*}$ a.s. and that $\mathbf{v}^{m}$ converges to $\mathbf{v}$ in $C\left(\left[t_{0}, t_{0}+T\right] ; U\right)$ [39]. Moreover $\mathbf{v}^{m}$ is regular and satisfies

$$
\mathrm{d} \mathbf{v}^{m}=\left(A \mathbf{v}^{m}+\mathbf{C} \mathbf{f}\left(\mathbf{v}^{m}+\mathbf{W}_{A}^{m}\right)+\mathbf{I}\right) \mathrm{d} t .
$$

Taking the inner product of (40) with $\mathbf{v}^{m}$ in $U$ and employing the result of (11), we find that

$$
\begin{aligned}
\frac{1}{2} \frac{\mathrm{d}}{\mathrm{d} t}\left\|\mathbf{v}^{m}\right\|^{2}= & \left(\mathbf{v}^{m}, \frac{\mathrm{d} \mathbf{v}^{m}}{\mathrm{~d} t}\right) \\
= & \left(\mathbf{v}^{m}, A \mathbf{v}^{m}+\mathbf{C f}\left(\mathbf{v}^{m}+\mathbf{W}_{A}^{m}\right)+\mathbf{I}\right) \\
\leq & -\left(\alpha \beta^{2}+k_{4}\right)\left\|\mathbf{v}^{m}\right\|^{2} \\
& +\left(\mathbf{v}^{m}, \mathbf{C f}\left(\mathbf{v}^{m}+\mathbf{W}_{A}^{m}\right)\right)+\left(\mathbf{v}^{m}, \mathbf{I}\right) .
\end{aligned}
$$

By the Cauchy-Schwartz inequality and Young inequality, we get

$$
\left(\mathbf{v}^{m}, \mathbf{I}\right) \leq\left\|\mathbf{v}^{m}\right\|\|\mathbf{I}\| \leq \frac{1}{2}\left\|\mathbf{v}^{m}\right\|^{2}+\frac{1}{2}\|\mathbf{I}\|^{2} .
$$

By using Young inequality and Proposition 3, as well as condition (H1), one obtains

$$
\begin{aligned}
& \left(\mathbf{v}^{m}, \mathbf{C} \mathbf{f}\left(\mathbf{v}^{m}+\mathbf{W}_{A}^{m}\right)\right) \leq\left\|\mathbf{v}^{m}\right\|\left\|\mathbf{C} \mathbf{f}\left(\mathbf{v}^{m}+\mathbf{W}_{A}^{m}\right)\right\| \\
& \leq \sqrt{n}\|\mathbf{C}\|_{F}\left\|\mathbf{v}^{m}\right\|\left\|\mathbf{f}\left(\mathbf{v}^{m}+\mathbf{W}_{A}^{m}\right)\right\| \\
& \leq \frac{1}{2} \sqrt{n}\|\mathbf{C}\|_{F}\left(\left\|\mathbf{v}^{m}\right\|^{2}+\left\|\mathbf{f}\left(\mathbf{v}^{m}+W_{A}^{m}\right)\right\|^{2}\right) \\
& \leq \frac{1}{2} \sqrt{n}\|\mathbf{C}\|_{F}\left(\left\|\mathbf{v}^{m}\right\|^{2}+2 k_{1}^{2}+2 k_{2}^{2}\left\|\mathbf{v}^{m}+\mathbf{W}_{A}^{m}\right\|^{2}\right) \\
& \leq \frac{1}{2} \sqrt{n}\|\mathbf{C}\|_{F}\left(\left(4 k_{2}^{2}+1\right)\left\|\mathbf{v}^{m}\right\|^{2}+2 k_{1}^{2}+4 k_{2}^{2}\left\|\mathbf{W}_{A}^{m}\right\|^{2}\right) .
\end{aligned}
$$

By (H2) and (H3), we deduce that

$$
\begin{aligned}
\frac{\mathrm{d}}{\mathrm{d} t} & \left\|\mathbf{v}^{m}\right\|^{2} \\
\leq & -2\left(\alpha \beta^{2}+k_{4}\right)\left\|\mathbf{v}^{m}\right\|^{2} \\
& +\sqrt{n}\|\mathbf{C}\|_{F}\left(\left(4 k_{2}+1\right)\left\|\mathbf{v}^{m}\right\|^{2}+2 k_{1}^{2}+4 k_{2}^{2}\left\|\mathbf{W}_{A}^{m}\right\|^{2}\right) \\
& +\left\|\mathbf{v}^{m}\right\|^{2}+\|\mathbf{I}\|^{2} \leq-\eta\left\|\mathbf{v}^{m}\right\|^{2}+\kappa,
\end{aligned}
$$

where $\gamma=2 \alpha \beta^{2}+2 k_{4}-\sqrt{n}\|\mathbf{C}\|_{F}\left(4 k_{2}^{2}+1\right)-1$ and $\kappa=\|\mathbf{I}\|^{2}+$ $2 \sqrt{n}\|\mathbf{C}\|_{F} k_{1}^{2}+4 k_{2}^{2} \sqrt{n}\|\mathbf{C}\|_{F}\left\|\mathbf{W}_{A}^{m}\right\|^{2}$. By the classical Gronwall inequality, then we have

$$
\begin{aligned}
& \left\|\mathbf{v}^{m}(t)\right\|^{2} \leq e^{-\gamma t}\left\|\boldsymbol{\psi}^{m}\right\|^{2}+\int_{t_{0}}^{t} e^{-\gamma s} \kappa \mathrm{d} s \leq\left\|\boldsymbol{\psi}^{m}\right\|^{2} \\
& +e^{|\gamma| T}\left(\|\mathbf{I}\|^{2}+2 \sqrt{n}\|\mathbf{C}\|_{F} k_{1}^{2}+4 k_{2}^{2} \sqrt{n}\|\mathbf{C}\|_{F} \mu_{m, \infty}^{2}\right) \\
& \cdot T
\end{aligned}
$$

with $\mu_{m, \infty}=\sup _{t \in\left[t_{0}, t_{0}+T\right]}\left\|\mathbf{W}_{A}^{m}(t)\right\|$, for a.e. $\omega \in \Omega$.

Taking the limit as $m \rightarrow \infty$, we see that a.s.

$$
\|\mathbf{v}(t)\|^{2} \leq\|\psi\|^{2}+\delta .
$$

It follows that

$$
\|\mathbf{v}(t)\| \leq\|\psi\|+\sqrt{\delta}
$$

thus we complete the proof. 

15.

It is easy to derive the following from Theorems 13, 14, and

Theorem 16. Let $\phi$ be given which is $\mathscr{F}_{0}$-measurable and bounded in $U$, a.s.; then there exists a unique mild solution $\mathbf{u}$ of (2), with $\mathbf{u} \in C\left(\left[t_{0}, t_{0}+T\right] ; U\right), \forall T<\infty$.

\section{Existence of the Random Attractor}

We refer $\omega=\mathbf{W}(t)$ and $\Omega=\left\{\omega \in C\left(\mathbb{R}, \mathbb{R}^{n}\right) \mid \boldsymbol{\omega}(0)=\mathbf{0}\right\}$, with $\mathbb{P}$ being the product measure of two Wiener measures on the negative and positive parts of $\Omega$. In this case, there exists a Wiener shift $\theta_{t}$ defined as $\theta_{t} \omega(s)=\omega(t+s)-\omega(s), s, t \in \mathbb{R}$, which is an ergodic transformation.

Let $\mathbf{v}\left(t, \omega ; t_{0}, \psi\right)$ be the solution of (23) with $\mathbf{v}(0)=\psi$ and $\mathbf{u}\left(t, \omega ; t_{0}, \phi\right)$ is the mild solution of (1) with $\mathbf{u}(0)=\phi$. Then, from Theorem 15, we know that the map $\psi \rightarrow \mathbf{v}\left(t, \omega ; t_{0}, \psi\right)$ is continuous, by $\mathbf{v}(t)=\mathbf{u}(t)-\mathbf{W}_{A}(t)$; then one can define the maps $S(t, s ; \omega)$ and $\varphi(t, \omega)$ by

$$
\begin{aligned}
S\left(t, t_{0} ; \omega\right) \boldsymbol{\phi} & =\mathbf{v}\left(t, \omega ; t_{0}, \psi\right)+\mathbf{W}_{\mathbf{A}}(t, \omega), \\
\boldsymbol{\varphi}(t, \omega) \boldsymbol{\phi} & =S(t, 0 ; \omega) \boldsymbol{\phi}=\mathbf{v}(t, 0 ; \omega) \psi+\mathbf{W}_{\mathbf{A}}(t, \omega) .
\end{aligned}
$$

By the result of Theorem 15, $S$ is a continuous stochastic flow and $\varphi$ is a continuous RDS associated with (1).

4.1. Absorbing Sets in U. Let us define the operator as follows:

$$
\begin{aligned}
\mathscr{A}: \Pi(\mathscr{A}) & \in U \longrightarrow U \\
\mathscr{A} \mathbf{u} & =-\nabla \cdot(G(\mathbf{x}) \circ \nabla \mathbf{u})
\end{aligned}
$$

and $\Pi(\mathscr{A})=\Pi(A)=\left\{H^{2}(\mathcal{O})\right\}^{n} \cap\left\{H_{0}^{1}(\mathcal{O})\right\}^{n}$. We can infer from (10) and (49) that

$$
A \mathbf{u}=-\mathscr{A} \mathbf{u}-\mathbf{B u}
$$

The associated bilinear operator with $\mathscr{A}$ is defined as $a(\mathbf{u}, \mathbf{v})=$ $(\mathscr{A} \mathbf{u}, \mathbf{v})$. By utilizing the result of [2], we have

$$
a(\mathbf{u}, \mathbf{u})=\int_{\mathcal{O}} G:(\nabla \mathbf{u} \circ \nabla \mathbf{u}) \mathrm{d} x \geq \alpha\|\mathbf{u}\|^{2},
$$

where $\mathbf{A}: \mathbf{B}$ is the Frobenius inner product of two $n \times m$ matrixes, defined as $\mathbf{A}: \mathbf{B} \triangleq \sum_{i}^{n} \sum_{j}^{m} a_{i j} b_{i j}$.

Let $t_{0}<-1$ and $\psi \in U$ be given, and let $\mathbf{v}$ be the solution of equation (23). Taking the inner product of (22) with $\mathbf{v}$ in $U$ we find that

$$
\begin{aligned}
\frac{1}{2} \frac{\mathrm{d}}{\mathrm{d} t}\|\mathbf{v}\|^{2}= & \left(\mathbf{v}, \frac{\mathrm{d} \mathbf{v}}{\mathrm{d} t}\right)=(\mathbf{v}, A \mathbf{v})+\mathbf{C f}\left(\mathbf{v}+\mathbf{W}_{\mathbf{A}}\right)+\mathbf{I} \\
\leq & -a(\mathbf{v}, \mathbf{v})-k_{4}\|\mathbf{v}\|^{2}+\left(\mathbf{v}, \mathbf{C} \mathbf{f}\left(\mathbf{v}+\mathbf{W}_{\mathbf{A}}\right)\right) \\
& +(\mathbf{v}, \mathbf{I}) .
\end{aligned}
$$

By the Cauchy-Schwartz inequality and Young inequality

$$
(\mathbf{v}, \mathbf{I}) \leq\|\mathbf{v}\|\|\mathbf{I}\| \leq \frac{k_{4}}{4}\|\mathbf{v}\|^{2}+\frac{1}{k_{4}}\|\mathbf{I}\|^{2} .
$$

By using Young inequality and (H1)

$$
\begin{aligned}
\left(\mathbf{v}, \mathbf{C f}\left(\mathbf{v}+\mathbf{W}_{\mathbf{A}}\right)\right) \leq & \|\mathbf{v}\|\left\|\mathbf{C} \mathbf{f}\left(\mathbf{v}+\mathbf{W}_{\mathbf{A}}\right)\right\| \\
\leq & k_{5}\|\mathbf{v}\|\left\|\mathbf{f}\left(\mathbf{v}+\mathbf{W}_{\mathbf{A}}\right)\right\| \\
\leq & \frac{k_{4}}{4}\|\mathbf{v}\|^{2}+\frac{k_{5}^{2}}{k_{4}}\left\|\mathbf{f}\left(\mathbf{v}+\mathbf{W}_{\mathbf{A}}\right)\right\|^{2} \\
& \leq\left(\frac{k_{4}}{4}+\frac{4 k_{2}^{2} k_{5}^{2}}{k_{4}}\right)\|\mathbf{v}\|^{2} \\
& +\frac{2 k_{5}^{2}}{k_{4}}\left(k_{1}^{2}+2 k_{2}^{2}\left\|\mathbf{W}_{\mathbf{A}}\right\|^{2}\right) .
\end{aligned}
$$

We deduce from (51)-(54) that

$$
\frac{\mathrm{d}}{\mathrm{d} t}\|\mathbf{v}\|^{2}+2 a(\mathbf{v}, \mathbf{v}) \leq-\eta\|\mathbf{v}\|^{2}+P_{1}(t, \omega),
$$

where $\eta=k_{4}-2 k_{2}^{2} k_{5}^{2} / k_{4}>0$ and $P_{1}(t, \omega)=\left(2 k_{5}^{2} / k_{4}\right)\left(k_{1}+\right.$ $\left.2 k_{2}\left\|\mathbf{W}_{\mathbf{A}}\right\|^{2}\right)+\left(2 / k_{4}\right)\|\mathbf{I}\|^{2}$. By (H4), we know that $\eta>0$; using the classical Gronwall inequality, for $t_{0} \leq-1$, we have

$$
\begin{aligned}
\|\mathbf{v}(-1)\|^{2} \leq & e^{-\eta\left(-1-t_{0}\right)}\left\|\mathbf{v}\left(t_{0}\right)\right\|^{2} \\
& +\int_{t_{0}}^{-1} e^{\eta(-1-s)} P_{1}(s, \omega) \mathrm{d} s \\
= & e^{-\eta\left(-1-t_{0}\right)}\left\|\mathbf{u}\left(t_{0}\right)\right\|^{2} \\
& +e^{-\eta\left(-1-t_{0}\right)}\left\|\mathbf{W}_{\mathbf{A}}\left(t_{0}\right)\right\|^{2} \\
& +\int_{t_{0}}^{-1} e^{-\eta(-1-s)} P_{1}(s, \omega) \mathrm{d} s .
\end{aligned}
$$

Theorem 17. Under conditions (H1)-(H4), the RDS $\varphi$ defined by (48) admits a random absorbing set in $U$.

Proof. Let the random variable

$$
r_{1}^{2}(\omega)=2+\int_{-\infty}^{-1} e^{-\eta(-1-s)} P_{1}(s, \omega) \mathrm{d} s .
$$

By (8), we know that $P_{1}(s, \omega)$ has at most polynomial growth for $s \rightarrow-\infty$, so $r(\omega)$ is finite. On the other hand, $\left\|\mathbf{W}_{\mathbf{A}}\left(t_{0}\right)\right\|^{2}$ also has at most polynomial growth for $t_{0}$, as $t_{0}$ tending to $-\infty$, according to (8). So, we can choose $\bar{t}$ such that

$$
e^{-\eta\left(-1-t_{0}\right)}\left(\|\phi\|^{2}+\left\|\mathbf{W}_{\mathbf{A}}\left(t_{0}\right)\right\|^{2}\right) \leq 2, \quad t_{0} \leq \bar{t} .
$$

By (56), (57), and (58), for all $\rho>0$, there exists $t(\omega) \leq-1$, such that for all $t_{0} \leq t(\omega)$ and $\phi \in U$, with $\|\phi\| \leq \rho$, the following inequality is satisfied:

$$
\left\|\mathbf{v}\left(-1, \omega ; t_{0}, \psi\right)\right\|^{2} \leq r_{1}^{2}(\omega) .
$$

By (48) and (59), the stochastic flow of system (1) satisfies

$$
\left\|S\left(-1, t_{0} ; \omega\right) \phi\right\| \leq r_{1}(\omega)+\left\|\mathbf{W}_{\mathbf{A}}\left(t_{0}, \omega\right)\right\| \triangleq r_{2}(\omega) .
$$


By using the relationship between stochastic flow and RDS

$$
\begin{aligned}
\boldsymbol{\varphi}( & \left(t, \theta_{-t} \omega\right) B_{U}(\mathbf{0}, \rho)=S\left(t, 0 ; \theta_{-t} \omega\right) B_{U}(\mathbf{0}, \rho) \\
& =S(0,-t ; \omega) B_{U}(\mathbf{0}, \rho) \\
& =S(0,-1 ; \omega) S(-1,-t ; \omega) B_{U}(\mathbf{0}, \rho) \\
& \subset S(0,-1 ; \omega) B_{U}\left(\mathbf{0}, r_{2}(\omega)\right), \quad \forall \rho>0,
\end{aligned}
$$

where $B_{U}(\mathbf{0}, \rho)$ denotes the ball of $U$ centered at $\mathbf{0}$ with radius $\rho$, let $K_{1}(\omega)=S(0,-1 ; \omega) B_{U}\left(\mathbf{0}, r_{2}(\omega)\right)$; there exists $t_{B}(\omega)>0$ such that $t \geq t_{B}(\omega)>0$

$$
\boldsymbol{\varphi}\left(t, \theta_{-t} \omega\right) B_{U}(\mathbf{0}, \rho) \subset K_{1}(\omega), \quad \text { a.e. } \omega \in \Omega .
$$

By Definition 9, we get the conclusion.

We need the following auxiliary proposition in the following sections.

Theorem 18. There exists a random variable $r_{3}(\omega), r_{4}(\omega)>0$, such that, for all $\rho>0$, there exist $t(\omega) \leq-1$, such that for all $t_{0} \leq t(\omega)$ and all $\phi \in U$, with $\|\phi\| \leq \rho$, the solution $\mathbf{v}\left(t, \omega ; t_{0}, \psi\right)$ of system (22) satisfies the inequality

$$
\begin{array}{r}
\int_{-1}^{0}\left\|\mathbf{v}\left(s, \omega ; t_{0}, \psi\right)\right\|^{2} \mathrm{~d} s \leq r_{3}(\omega), \\
\int_{-1}^{0}\|a(\mathbf{v}, \mathbf{v})\| \mathrm{d} s \leq r_{4}(\omega) .
\end{array}
$$

Proof. From (55), we can get

$$
\int_{-1}^{0} a(\mathbf{v}, \mathbf{v}) \mathrm{d} s \leq \frac{1}{2}\|\mathbf{v}(-1)\|^{2}+\frac{1}{2} \int_{-1}^{0} P_{1}(s, \omega) \mathrm{d} s .
$$

Integrating (55) over $[-1,0]$, we can also get

$$
\int_{-1}^{0}\|\mathbf{v}\|^{2} \mathrm{~d} s \leq \frac{1}{\eta}\|\mathbf{v}(-1)\|^{2}+\frac{1}{\eta} \int_{-1}^{0} P_{1}(s, \omega) \mathrm{d} s .
$$

Let $r_{3}(\omega)=(1 / 2)\|\mathbf{v}(-1)\|^{2}+(1 / 2) \int_{-1}^{0} P_{1}(s, \omega) \mathrm{d} s, r_{4}(\omega)=$ $(1 / \eta)\|\mathbf{v}(-1)\|^{2}+(1 / \eta) \int_{-1}^{0} P_{1}(s, \omega) \mathrm{d} s$, by Theorem $17,\|\mathbf{v}(-1)\|$ is finite, and by using (8) again, we know that $r_{3}(\omega), r_{4}(\omega)$ is finite.

4.2. Absorbing Set in $V$. We now prove the existence of an absorbing set in $V$. Multiplying (22) by $\mathscr{A} \mathbf{v}$ and integrating over $\mathcal{O}$, by $(50)$, we have

$$
\begin{aligned}
\left(\mathscr{A} \mathbf{v}, \frac{\mathrm{d} \mathbf{v}}{\mathrm{d} t}\right)= & \left(\mathscr{A} \mathbf{v}, A \mathbf{v}+C \mathbf{f}\left(\mathbf{v}+\mathbf{W}_{\mathbf{A}}\right)+\mathbf{I}\right) \\
\leq & -\|\mathscr{A} \mathbf{v}\|^{2}-(\mathscr{A} \mathbf{v}, \mathbf{B v}) \\
& +\|\mathscr{A} \mathbf{v}\|\left\|\mathbf{C} \mathbf{f}\left(\mathbf{v}+\mathbf{W}_{\mathbf{A}}\right)\right\|+(\mathscr{A} \mathbf{v}, \mathbf{I}) .
\end{aligned}
$$

Using the Dirichlet boundary condition and the Green first identity

$$
\left(\mathscr{A} \mathbf{v}, \frac{\mathrm{d} \mathbf{v}}{\mathrm{d} t}\right)=a\left(\mathbf{v}, \frac{\mathrm{d}}{\mathrm{d} t} \mathbf{v}\right)=\frac{1}{2} \frac{\mathrm{d}}{\mathrm{d} t} a(\mathbf{v}, \mathbf{v}) .
$$

By Gauss formula [3]

$$
(\mathscr{A} \mathbf{v}, \mathbf{B v})=\int_{\mathscr{O}}(B G):(\nabla \mathbf{v} \circ \nabla \mathbf{v}) \mathrm{d} \mathbf{x} \geq k_{4} \alpha\|\mathbf{v}\|^{2} .
$$

We can also infer from the divergence theorem

$$
(\mathscr{A} \mathbf{v}, \mathbf{I})=-\sum_{i=1}^{n} \int_{\partial \mathcal{O}} I_{i}\left(G_{i} \nabla u_{i}\right) \cdot \mathrm{d} s=0 .
$$

Using the Young inequality again, we get

$$
\|\mathscr{A} \mathbf{v}\|\left\|\mathbf{C f}\left(\mathbf{v}+\mathbf{W}_{\mathbf{A}}\right)\right\| \leq\|\mathscr{A} \mathbf{v}\|^{2}+\frac{1}{4}\left\|\mathbf{C f}\left(\mathbf{v}+\mathbf{W}_{\mathbf{A}}\right)\right\|^{2}
$$

we can deduce from Proposition 3 and condition (H1)

$$
\begin{aligned}
& \left\|\mathbf{C} \mathbf{f}\left(\mathbf{v}+\mathbf{W}_{\mathbf{A}}\right)\right\|^{2} \leq n\|\mathbf{C}\|_{F}^{2}\left\|\mathbf{f}\left(\mathbf{v}+\mathbf{W}_{\mathbf{A}}\right)\right\|^{2} \\
& \quad \leq n\|\mathbf{C}\|_{F}^{2}\left(4 k_{2}^{2}\|\mathbf{v}\|^{2}+2 k_{1}^{2}+4 k_{2}^{2}\left\|\mathbf{W}_{\mathbf{A}}\right\|^{2}\right) .
\end{aligned}
$$

It follows from (66)-(71) that

$$
\frac{\mathrm{d}}{\mathrm{d} t} a(\mathbf{v}, \mathbf{v}) \leq-k_{9} a(\mathbf{v}, \mathbf{v})+P_{2}(t, \omega)
$$

where $k_{9}=2 k_{4}$

$$
P_{2}(t, \omega)=n\|\mathbf{C}\|_{F}^{2}\left(2 k_{2}^{2}\|\mathbf{v}\|^{2}+k_{1}^{2}+2 k_{2}^{2}\left\|\mathbf{W}_{\mathbf{A}}\right\|^{2}\right) .
$$

Integrating (72) over $[s, 0]$, we have

$$
a(\mathbf{v}(0), \mathbf{v}(0)) \leq a(\mathbf{v}(s), \mathbf{v}(s))+\int_{s}^{0} P_{2}(s, \omega) \mathrm{d} s
$$

integrating (72) again in $s$ over $[-1,0]$ and using (51), we have

$$
\begin{aligned}
\|\mathbf{v}(0)\|^{2} \leq & \frac{1}{\alpha} \int_{-1}^{0} a(\mathbf{v}(s), \mathbf{v}(s)) \mathrm{d} s \\
& +\frac{1}{\alpha} \int_{-1}^{0} P_{2}(s, \omega) \mathrm{d} s .
\end{aligned}
$$

Theorem 19. Under conditions (H1)-(H4), the RDS $\varphi$ defined by (48) admits an absorbing set in $V$.

Proof. Put the random variable

$$
r_{5}^{2}(\omega) \triangleq \frac{1}{\alpha} \int_{-1}^{0} a(\mathbf{v}(s), \mathbf{v}(s)) \mathrm{d} s+\frac{1}{\alpha} \int_{-1}^{0} P_{2}(s, \omega) \mathrm{d} s .
$$

By Theorem 18, $r_{5}$ is a finite number; then by employing (71), for all $\rho>0$, there exist $t(\omega) \leq-1$, for all $t_{0} \leq t(\omega)$ and all $\phi \in V$, with $\|\phi\| \leq \rho$; the solution $\mathbf{v}\left(0, \omega ; t_{0}, \psi\right)$ of system (23) satisfies the inequality

$$
\left\|\mathbf{v}\left(0, \omega ; t_{0}, \phi-\mathbf{W}_{\mathbf{A}}(0, \omega)\right)\right\|^{2} \leq r_{5}^{2}(\omega)
$$

which also means the stochastic flow of (1) satisfies

$$
\left\|S\left(0, t_{0} ; \omega\right) \boldsymbol{\phi}\right\| \leq r_{5}(\omega)+\left\|\mathbf{W}_{\mathbf{A}}\left(t_{0}, \omega\right)\right\| \triangleq r_{6}(\omega) .
$$



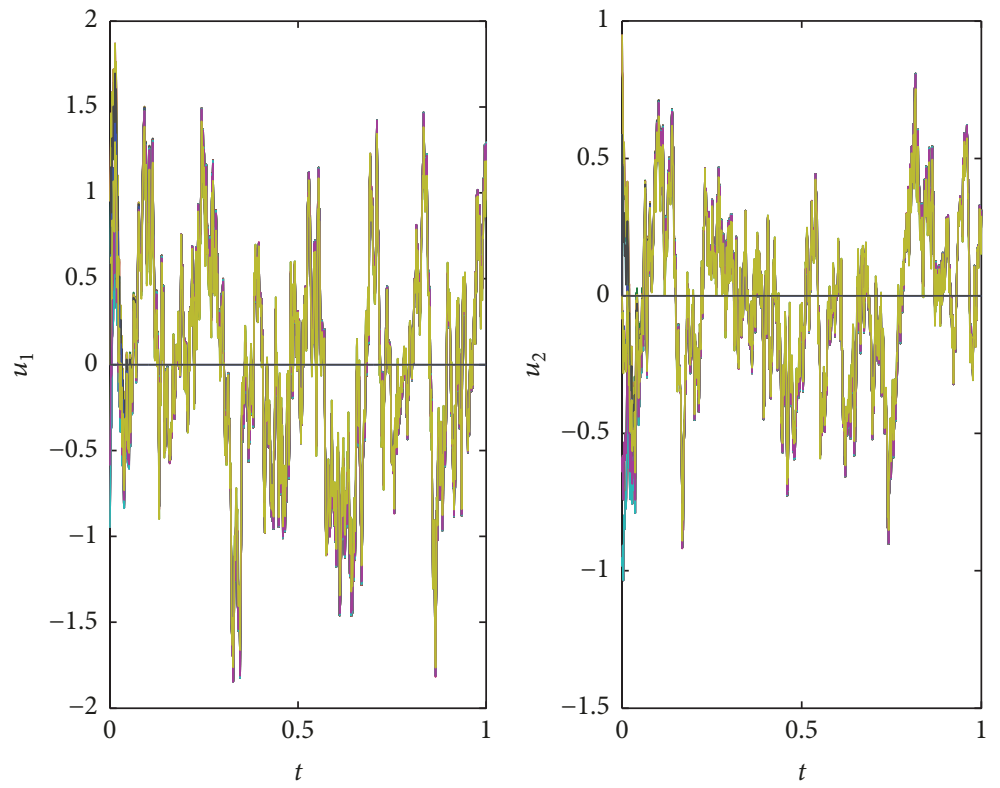

FIGURE 1: Frequency of Example 1.

By using the definition of RDS and the stochastic flow, we have

$$
\varphi\left(t, \theta_{-t} \omega\right)=S\left(t, 0 ; \theta_{-t} \omega\right)=S(0,-t ; \omega)
$$

By (78) and (79), $\forall\|\phi\| \leq \rho$, for sufficiently large $t$

$$
\left\|\varphi\left(t, \theta_{-t} \omega\right) \phi\right\| \leq r_{6}(\omega)
$$

Let $K_{2}(\omega)=B_{V}\left(\mathbf{0}, r_{6}(\omega)\right)$, where $B_{V}\left(\mathbf{0}, r_{6}\right)$ denotes the ball of $V$ centered at $\mathbf{0}$ with radius $r_{6}$, so for all $B_{V}(\mathbf{0}, \rho) \in U$, there exists $t_{B}(\omega)>0$ such that $t \geq t_{B}(\omega)>0$

$$
\boldsymbol{\varphi}\left(t, \theta_{-t} \omega\right) B_{V}(0, \rho) \subset K_{2}(\omega), \quad \text { a.e. } \omega \in \Omega
$$

Theorem 20. Assume that (H1)-(H4) hold; then the RDS $\varphi$ generated by (1) possesses a random attractor in $U$.

Proof. By Theorem 19, $\varphi(t ; \omega) \phi$ is absorbed by the random set $K_{2}(\omega)$. Since the embedding of $V$ to $U$ is compact, so the bounded set $K_{2}(\omega)$ is compact. A combination of Lemma 11 and Theorems 17 and 19, we get the existence of random attractor for the $\operatorname{RDS} \varphi$.

If the active function $\mathbf{f}$ is a bounded Lipschitz function, then we can choose a sufficiently large $k_{1}$ such that $k_{2}=0$, so (H4) is satisfied automatically; then we get the following theorem.

Corollary 21. Under conditions (H1)-(H3) and the fact that there exists a constant $k_{1}$ such that $\|\mathbf{f}(\mathbf{u})\| \leq k_{1}$, the RDS $\boldsymbol{\varphi}$ defined by (48) admits a random attractor in $U$.

\section{Example and Simulation}

Example 1.

$$
\begin{aligned}
\dot{u}_{1}(t, \mathbf{x})= & \Delta u_{1}-2 u_{1}-2 \tanh \left(u_{1}(t-1, x)\right) \\
& -\tanh \left(u_{2}(t-1, x)\right)+1.3 \frac{\partial^{2} W}{\partial t \partial x}, \\
\dot{u}_{2}(t, \mathbf{x})= & \Delta u_{2}-2 u_{2}+\tanh \left(u_{1}(t-1, x)\right) \\
& -3 \tanh \left(u_{2}(t-1, x)\right)+1.5 \frac{\partial^{2} W}{\partial t \partial x}, \\
u_{i}(t, 0)= & u_{i}(t, 20)=0, \quad t \geq 0, \\
u_{1}(0, \mathbf{x})= & \sin (0.2 \pi \mathbf{x}), \\
u_{2}(0, \mathbf{x})= & \sin (0.2 \pi \mathbf{x}),
\end{aligned}
$$

$$
x \in \mathcal{O}=[0,20] .
$$

Proof. In this case $U=\left\{L^{2}(\mathcal{O})\right\}^{2}$ and $\mathbf{f}(\mathbf{u})$ is a bounded global Lipschitz continuous function with $k_{1}=20, k_{2}=0$, and $k_{3}=1,\|S(t)\| \leq 1$, and $\Delta$ is the Laplace operator. $E W=0$ and $W$ is the cylindrical Wiener process with $E[W(s$, $x) \wedge W(t, y)]=(t \wedge s)(x \wedge y), \forall s, t \geq 0, x, y \in[0,20]$, $E$ is the expectation, and $a \wedge b=\min \{a, b\}$. Under these assumptions, by Theorem 15 , this system has a global mild solution. Meanwhile $k_{4} \geq 2 \sqrt{2} k_{2} k_{5}$, so according to Theorem 20 , this system possesses a random attractor.

We simulate this example by using the Matlab; for detailed information see Figures 1-3. A Crank-Nicolson method in time and second-order center differences in space are used to discrete this model. A Newton iterative method is used to solve the discretized nonlinear equation. For more theories 


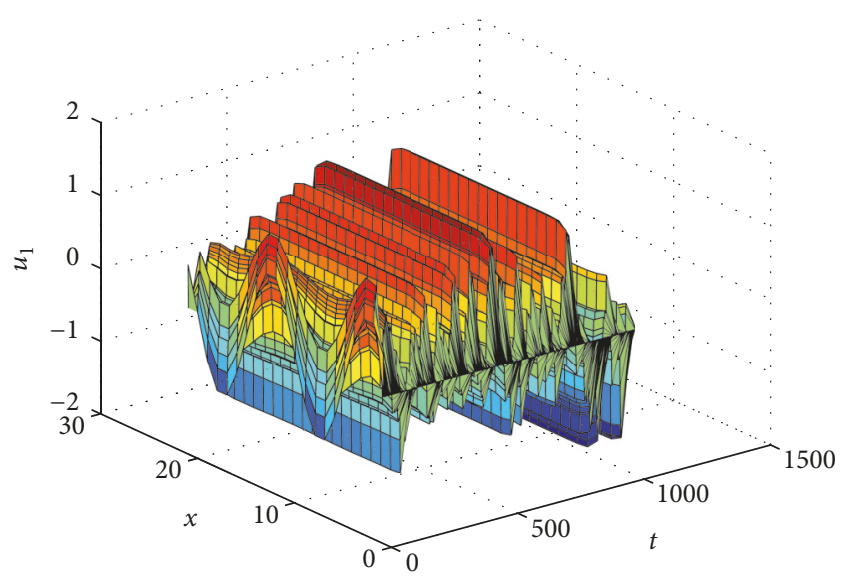

FIGURE 2: Simulation of $u_{1}$ in Example 1.

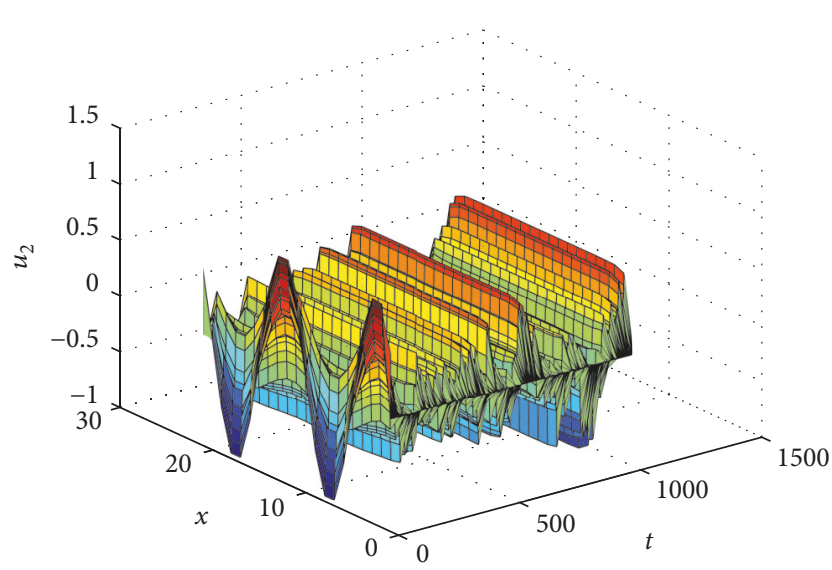

Figure 3: Simulation of $u_{2}$ in Example 1.

about the numerical theory about SPDE, we refer to [42, 43].

The method used in this article can be extended to other systems, such as the biological systems and fluid mechanical systems [44-48]. We can also apply this method to the system driven by other types of noise, such as the G-Brown motion [49]. By the way, some control technique may be used to stabilize these systems with random attractor [50-54]. We will study these problems in the future.

\section{Conflicts of Interest}

The authors declare that they have no conflicts of interest.

\section{Acknowledgments}

The work was supported in part by the National Natural Science Foundation of China under Grant 11372051, Grant 11201035, and Grant 11475029, in part by the Fund of the China Academy of Engineering Physics under Grant 2015B0202045, in part by the Shandong Provincial Natural Science Foundation under Grant ZR2017BA014, and in part by Development Program for Defense Department of China under Grant C1520110002.

\section{References}

[1] L. Wang and D. Xu, "Global exponential stability of Hopfield reaction-diffusion neural networks with time-varying delays," Science in China, Series F: Information Sciences, vol. 46, no. 6, pp. 466-474, 2003.

[2] X. Liang, L. Wang, Y. Wang, and R. Wang, "Dynamical behavior of delayed reaction-diffusion Hopfield neural networks driven by infinite dimensional Wiener processes," IEEE Transactions on Neural Networks and Learning Systems, vol. 27, no. 9, pp. 1816-1826, 2016.

[3] L. Wang, R. Zhang, and Y. Wang, "Global exponential stability of reaction-diffusion cellular neural networks with S-type distributed time delays," Nonlinear Analysis: Real World Applications, vol. 10, no. 2, pp. 1101-1113, 2009.

[4] L. Wang, Delayed Recurrent Neural Networks, Science Press, Beijing, China, 2008.

[5] A. N. Michel and D. Liu, Qualitative Analysis and Synthesis of Recurrent Neural Networks, vol. 248 of Monographs and Textbooks in Pure and Applied Mathematics, Marcel Dekker, New York, NY, USA, 2002.

[6] L. Wang and Y. Gao, "Global exponential robust stability of reaction-diffusion interval neural networks with time-varying delays," Physics Letters A, vol. 350, no. 5-6, pp. 342-348, 2006.

[7] Q. Zhu, X. Li, and X. Yang, "Exponential stability for stochastic reaction-diffusion BAM neural networks with time-varying and distributed delays," Applied Mathematics and Computation, vol. 217, no. 13, pp. 6078-6091, 2011.

[8] X. Liao, Y. Fu, J. Gao, and X. Zhao, "Stability of Hopfield neural networks with reaction-diffusion terms," Acta Electronica Sinica, vol. 28, no. 1, pp. 78-80, 2000.

[9] H. Zhao and G. Wang, "Existence of periodic oscillatory solution of reaction-diffusion neural networks with delays," Physics Letters A, vol. 343, no. 5, pp. 372-383, 2005.

[10] L. O. Chua, "Passivity and complexity," IEEE Transactions on Circuits and Systems I: Fundamental Theory and Applications, vol. 46, no. 1, pp. 71-82, 1999.

[11] R. Guo, Z. Zhang, X. Liu, and C. Lin, "Existence, uniqueness, and exponential stability analysis for complex-valued memristor-based BAM neural networks with time delays," Applied Mathematics and Computation, vol. 311, pp. 100-117, 2017.

[12] L. Wang, Z. Zhang, and Y. Wang, "Stochastic exponential stability of the delayed reaction-diffusion recurrent neural networks with Markovian jumping parameters," Physics Letters A, vol. 372, no. 18, pp. 3201-3209, 2008.

[13] J. Sun and L. Wan, "Convergence dynamics of stochastic reaction-diffusion recurrent neural networks with delays," International Journal of Bifurcation and Chaos, vol. 15, no. 7, pp. 21312144, 2005.

[14] G. da Prato and J. Zabczyk, Stochastic Equations in Infinite Dimensions, vol. 44, Cambridge University Press, Cambridge, UK, 1992.

[15] H.-J. Ma and T. Hou, "A separation theorem for stochastic singular linear quadratic control problem with partial information," Acta Mathematicae Applicatae Sinica, vol. 29, no. 2, pp. 303-314, 2013.

[16] Z. Yan and W. Zhang, "Finite-time stability and stabilization of itô-type stochastic singular systems," Abstract and Applied Analysis, vol. 2014, Article ID 263045, 9 pages, 2014. 
[17] H. Ma and Y. Jia, "Stability analysis for stochastic differential equations with infinite Markovian switchings," Journal of Mathematical Analysis and Applications, vol. 435, no. 1, pp. 593-605, 2016.

[18] X. Meng and X. Wang, "Stochastic predator-prey system subject to lévy jumps," Discrete Dynamics in Nature and Society, vol. 2016, Article ID 5749892, 13 pages, 2016.

[19] T. Caraballo, I. D. Chueshov, and P. E. Kloeden, "Synchronization of a stochastic reaction-diffusion system on a thin twolayer domain," SIAM Journal on Mathematical Analysis, vol. 38, no. 5, pp. 1489-1507, 2006/07.

[20] R. Temam, Infinite Dimensional Dynamical Systems in Mechanics and Physics, vol. 68, Springer, NY, USA, 1988.

[21] J. Duan, K. Lu, and B. Schmalfuss, "Invariant manifolds for stochastic partial differential equations," Annals of Probability, vol. 31, no. 4, pp. 2109-2135, 2003.

[22] L. Arnold, Random Dynamical Systems, Springer Monographs in Mathematics, Springer-Verlag, Berlin, Germany, 1998.

[23] Z. a. Brzézniak and Y. Li, "Asymptotic compactness and absorbing sets for 2D stochastic Navier-Stokes equations on some unbounded domains," Transactions of the American Mathematical Society, vol. 358, no. 12, pp. 5587-5629, 2006.

[24] P. W. Bates, K. Lu, and B. Wang, "Random attractors for stochastic reaction-diffusion equations on unbounded domains," Journal of Differential Equations, vol. 246, no. 2, pp. 845-869, 2009.

[25] D. Yang, "Random attractors for the stochastic KuramotoSivashinsky equation," Stochastic Analysis and Applications, vol. 24, no. 6, pp. 1285-1303, 2006.

[26] H. Kunita, Stochastic Flows and Stochastic Differential Equations, vol. 24 of Cambridge Studies in Advanced Mathematics, Cambridge University Press, London, UK, 1990.

[27] B. Gess, W. Liu, and M. Röckner, "Random attractors for a class of stochastic partial differential equations driven by general additive noise," Journal of Differential Equations, vol. 251, no. 45, pp. 1225-1253, 2011.

[28] F. Flandoli and B. Schmalfuss, "Random attractors for the $3 \mathrm{D}$ stochastic navier-stokes equation with multiplicative white noise," Stochastics and Stochastics Reports, vol. 59, no. 1-2, pp. 21-45, 1996.

[29] T. Caraballo, J. A. Langa, and J. C. Robinson, "A stochastic pitchfork bifurcation in a reaction-diffusion equation," Proceedings of the Royal Society of London. Series A, vol. 457, no. 2013, pp. 20412061, 2001.

[30] Z. Wang and S. Zhou, "Random attractor for stochastic reaction-diffusion equation with multiplicative noise on unbounded domains," Journal of Mathematical Analysis and Applications, vol. 384, no. 1, pp. 160-172, 2011.

[31] M. Hairer and J. C. Mattingly, "Ergodicity of the 2D NavierStokes equations with degenerate stochastic forcing," Annals of Mathematics: Second Series, vol. 164, no. 3, pp. 993-1032, 2006.

[32] P. E. Kloeden and J. A. Langa, "Flattening, squeezing and the existence of random attractors," Proceedings of the Royal Society A Mathematical, Physical and Engineering Sciences, vol. 463, no. 2077, pp. 163-181, 2007.

[33] J. K. Hale and S. M. V. Lunel, Introduction to FunctionalDifferential Equations, Springer, Berlin, Germany, 1993.

[34] P. Balasubramaniam, S. K. Ntouyas, and D. Vinayagam, "Existence of solutions of semilinear stochastic delay evolution inclusions in a Hilbert space," Journal of Mathematical Analysis and Applications, vol. 305, no. 2, pp. 438-451, 2005.
[35] T. Zhang, X. Meng, Y. Song, and T. Zhang, "A stage-structured predator-prey SI model with disease in the prey and impulsive effects," Mathematical Modelling and Analysis, vol. 18, no. 4, pp. 505-528, 2013.

[36] T. Zhang, X. Meng, T. Zhang, and Y. Song, "Global dynamics for a new high-dimensional SIR model with distributed delay," Applied Mathematics and Computation, vol. 218, no. 24, pp. 11806-11819, 2012.

[37] Y. Cui, "Uniqueness of solution for boundary value problems for fractional differential equations," Applied Mathematics Letters, vol. 51, pp. 48-54, 2016.

[38] Z. Bai, X. Dong, and C. Yin, "Existence results for impulsive nonlinear fractional differential equation with mixed boundary conditions," Boundary Value Problems, vol. 2016, no. 1, article no. 63, 2016.

[39] H. Crauel and F. Flandoli, "Attractors for random dynamical systems," Probability Theory and Related Fields, vol. 100, no. 3, pp. 365-393, 1994.

[40] H. Crauel, A. Debussche, and F. Flandoli, "Random attractors," Journal of Dynamics and Differential Equations, vol. 9, no. 2, pp. 307-341, 1997.

[41] G. Da Prato, A. Debussche, and R. Temam, "Stochastic Burgers' equation," Nonlinear Differential Equations and Applications NoDEA, vol. 1, no. 4, pp. 389-402, 1994.

[42] H. Choi, R. Temam, P. Moin, and J. Kim, "Feedback control for unsteady flow and its application to the stochastic burgers equation," Journal of Fluid Mechanics, vol. 253, pp. 509-543, 1993.

[43] S. Zhang, X. Meng, T. Feng, and T. Zhang, "Dynamics analysis and numerical simulations of a stochastic non-autonomous predator-prey system with impulsive effects," Nonlinear Analysis: Hybrid Systems, vol. 26, pp. 19-37, 2017.

[44] T. Zhang, X. Meng, and T. Zhang, "Global dynamics of a virus dynamical model with cell-to-cell transmission and cure rate," Computational and Mathematical Methods in Medicine, vol. 2015, Article ID 758362, 8 pages, 2015.

[45] Y. Zhang, K. Lv, S. Wang, J. Su, and D. Meng, "Modeling gene networks in saccharomyces cerevisiae based on gene expression profiles," Computational and Mathematical Methods in Medicine, vol. 2015, Article ID 621264, 10 pages, 2015.

[46] X. Zhang, L. Liu, Y. Wu, and Y. Cui, "Entire blow-up solutions for a quasilinear $\mathrm{p}$-Laplacian Schrodinger equation with a nonsquare diffusion term," Applied Mathematics Letters, vol. 74, no. 1, pp. 85-93, 2017.

[47] X. Zhou, X. Shi, and H. Cheng, "Modelling and stability analysis for a tuberculosis model with healthy education and treatment," Computational \& Applied Mathematics, vol. 32, no. 2, pp. 245260, 2013.

[48] T. Zhang, X. Meng, and T. Zhang, "SVEIRS: a new epidemic disease model with time delays and impulsive effects," Abstract and Applied Analysis, vol. 2014, Article ID 542154, 15 pages, 2014.

[49] X. Li, X. Lin, and Y. Lin, "Lyapunov-type conditions and stochastic differential equations driven by G-Brownian motion," Journal of Mathematical Analysis and Applications, vol. 439, no. 1, pp. 235-255, 2016.

[50] M. Gao, L. Sheng, and W. Zhang, "Stochastic H-2/H-infinity control of nonlinear systems with time-delay and state-dependent noise," Applied Mathematics and Computation, vol. 266, no. 2, pp. 429-440, 2015.

[51] G. Li, C. Ding, and M. Chen, "Intertwined basins of attraction of dynamical systems," Applied Mathematics and Computation, vol. 213, no. 1, pp. 272-274, 2009. 
[52] Z. Zhang, H. Shao, Z. Wang, and H. Shen, "Reduced-order observer design for the synchronization of the generalized Lorenz chaotic systems," Applied Mathematics and Computation, vol. 218, no. 14, pp. 7614-7621, 2012.

[53] G. Li and W. Zhang, "Study on indefinite stochastic linear quadratic optimal control with inequality constraint," Journal of Applied Mathematics, vol. 2013, Article ID 805829, 9 pages, 2013.

[54] Z. Yan, G. Zhang, J. Wang, and W. Zhang, "State and output feedback finite-time guaranteed cost control of linear Ito stochastic systems," Journal of Systems Science \& Complexity, vol. 28, no. 4, pp. 813-829, 2015. 


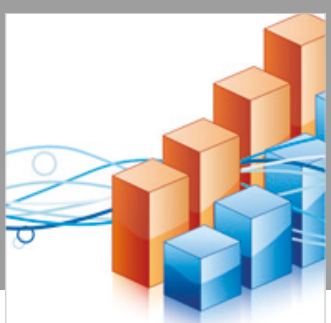

Advances in

Operations Research

\section{-n-m}
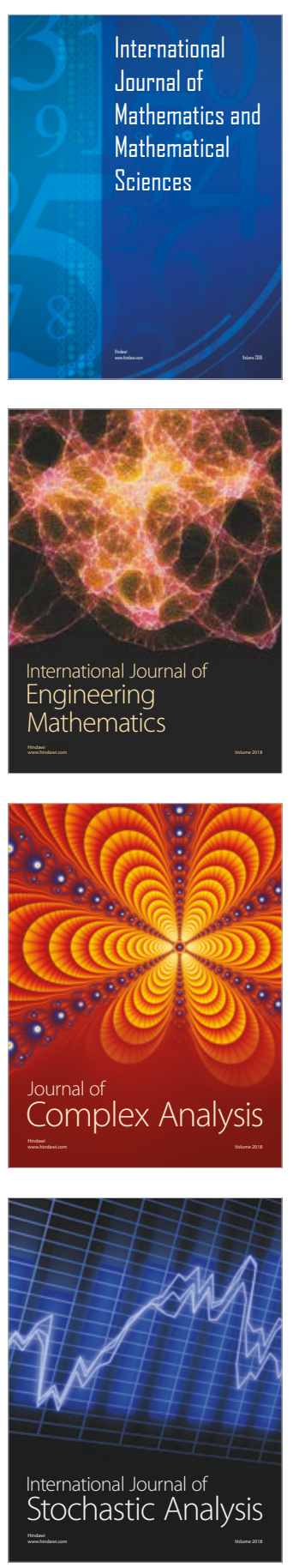
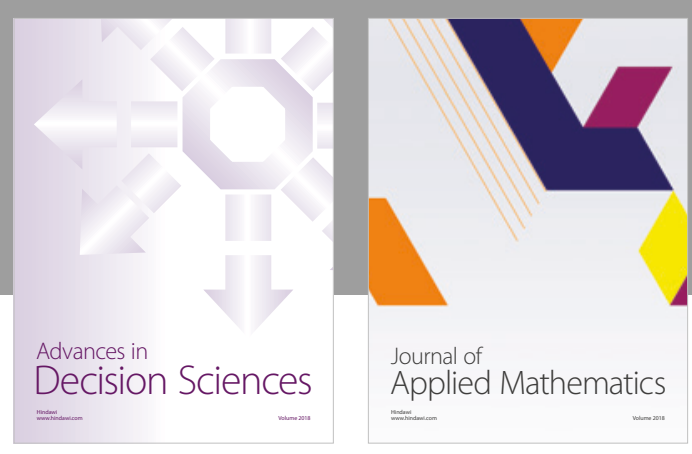

Journal of

Applied Mathematics
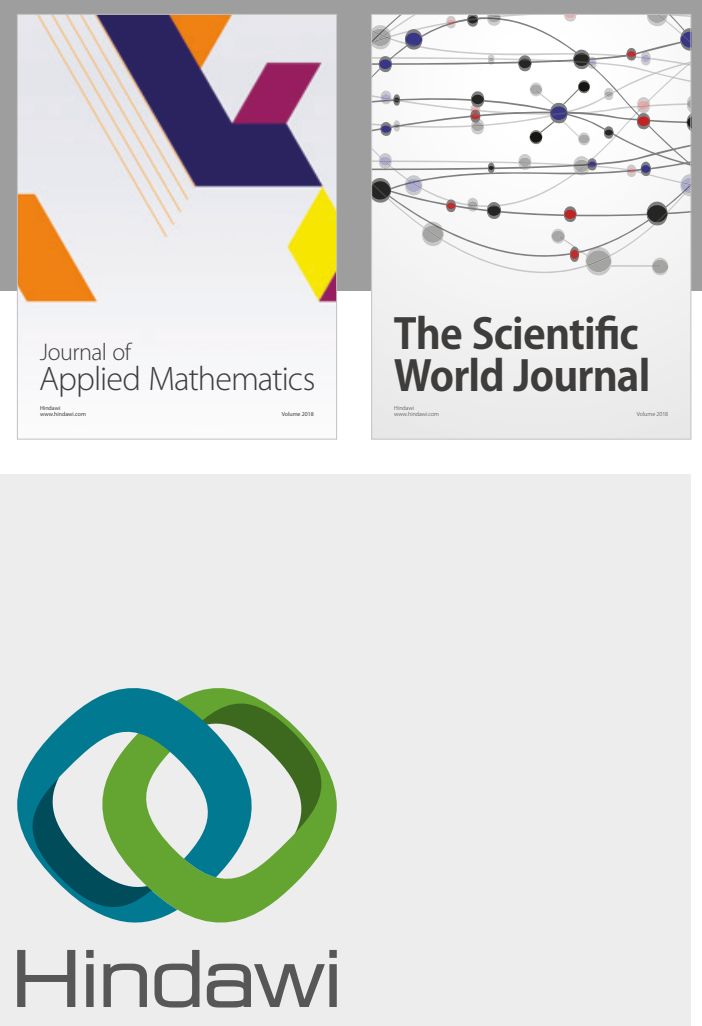

Submit your manuscripts at

www.hindawi.com

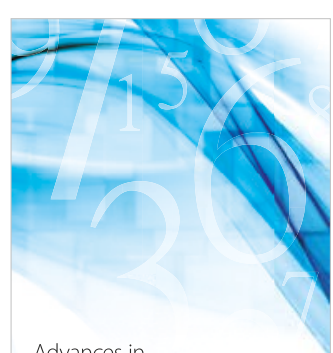

Advances in
Numerical Analysis
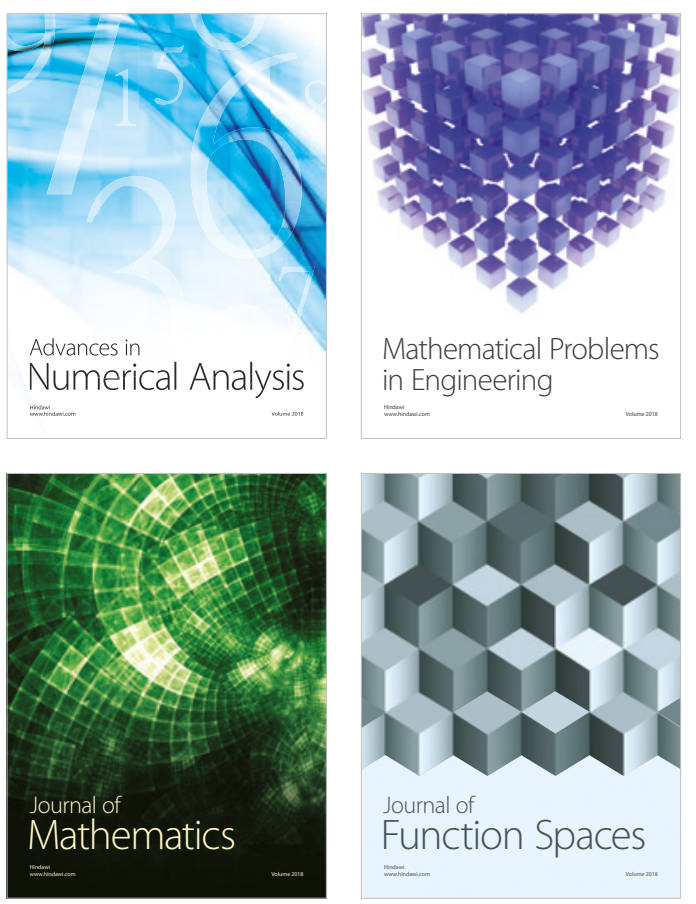

Mathematical Problems in Engineering

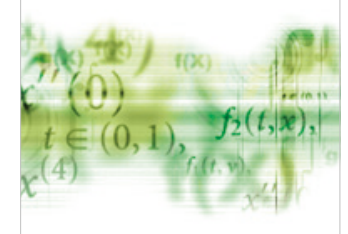

International Journal of

Differential Equations

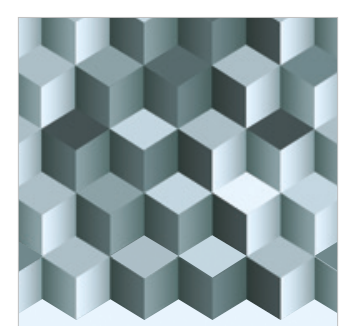

Journal of

Function Spaces

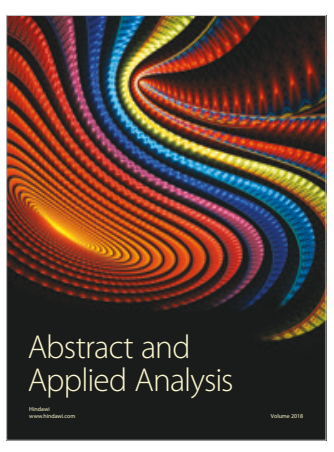

The Scientific

World Journal

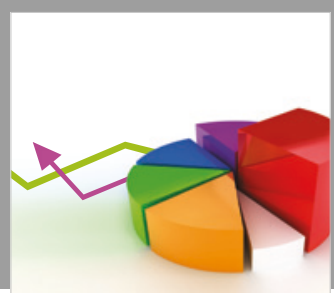

Journal of

Probability and Statistics
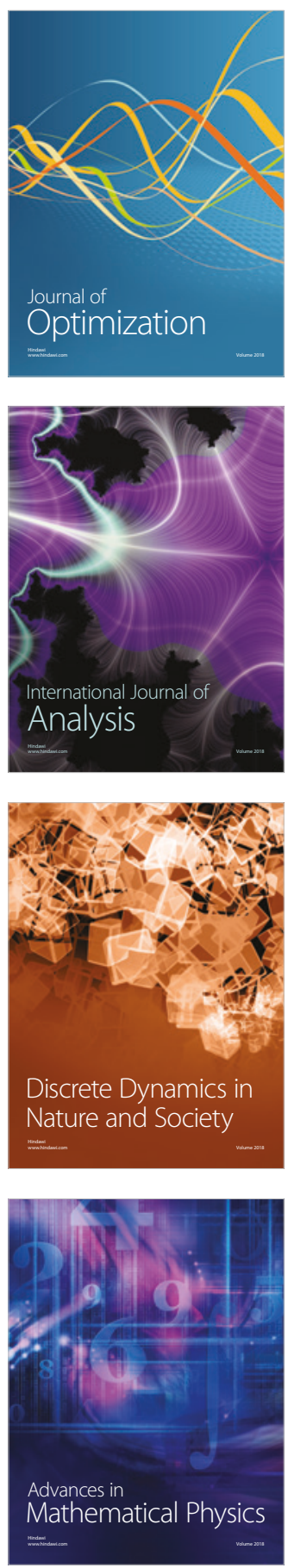